\title{
Bifurcation Analysis of a Stage-Structured Prey-Predator System with Discrete and Continuous Delays
}

\author{
Shunyi Li, Wenwu Liu, Xiangui Xue \\ Department of Mathematics, Qiannan Normal College for Nationalities, Duyun, China \\ Email: lishunyi19820425@163.com
}

Received May 5, 2013; revised June 5, 2013; accepted June 14, 2013

Copyright (C) 2013 Shunyi Li et al. This is an open access article distributed under the Creative Commons Attribution License, which permits unrestricted use, distribution, and reproduction in any medium, provided the original work is properly cited.

\begin{abstract}
A three-stage-structured prey-predator model with discrete and continuous time delays is studied. The characteristic equations and the stability of the boundary and positive equilibrium are analyzed. The conditions for the positive equilibrium occurring Hopf bifurcation are given, by applying the theorem of Hopf bifurcation. Finally, numerical simulation and brief conclusion are given.
\end{abstract}

Keywords: Three-Stage-Structured; Prey-Predator Model; Time Delay; Hopf Bifurcation

\section{Introduction}

In the natural world, there are many species whose individual members have a life history that takes them through two stages: immature and mature. In 1990, Aiello and Freedman [1] introduced single-species stagestructured model with time delay, and the stability of the system was studied. In 1997, Wang and Chen [2] introduced single-species stage-structured model without time delay and found that an orbitally asymptotically stable periodic orbit existence. In these papers [3], the authors assume that the life history of each population is divided into distinctive stages: the immature and mature members of the population, where only the mature member can reproduce themselves. However, in the nature many species go through three life stages: immature, mature and old. For example, many female animals lose reproductive ability when they are old.

A single species with three life history stage and cannibalism model have considered by S. J. Gao [4], and shown that the stability of the positive equilibrium can change a finite number of times at most as time delay is increased when the model under some parameters values. Recently, a nonautonomous three-stage-structured predator-prey system with time delay have studied by S. J. Yang and B. Shi [5], by using the continuation theorem of coincidence degree theory, the existence of a positive periodic solution is obtained. And the local Hopf bifurcation and global periodic solutions for a delayed threestage-structured predator-prey considered by Li et al. [6, 7].

\section{Formulation of the Model}

In this paper, we consider following three-stage-structured prey-predator model with discrete and continuous time delays

$$
\left\{\begin{array}{l}
x_{1}^{\prime}(t)=\alpha x_{2}(t)-x_{1}(t)\left(\gamma_{1}-\Omega-\eta x_{1}(t)-E y(t)\right), \\
x_{2}^{\prime}(t)=\Omega x_{1}(t)-x_{2}(t)\left(\theta_{1}+a\right), \\
x_{3}^{\prime}(t)=a x_{2}(t)-b x_{3}(t), \\
y^{\prime}(t)=y(t)\left[k E x(t-\tau)-d-f \int_{-\infty}^{t} \delta y\left(\tau_{1}\right) \mathrm{e}^{-\delta\left(t-\tau_{1}\right)} \mathrm{d} \tau_{1}\right]
\end{array}\right.
$$

where

$$
\int_{-\infty}^{t} \delta \mathrm{e}^{-\delta\left(t-\tau_{1}\right)} \mathrm{d} \tau_{1}=\int_{0}^{+\infty} \delta \mathrm{e}^{-\delta s} \mathrm{~d} s=1,
$$

$x_{1}(t), x_{2}(t), x_{3}(t)$ are the densities of immature preys, mature preys and old preys population at time $t, y(t)$ is the density of predator population at time $t$, respectively. All of the parameters are positive, $\alpha$ is the birth rate of mature prey population, and $\gamma_{1}, \theta_{1}, b$ are the death rate of immature, mature and old prey population, respectively. $\Omega$ and $a$ are the maturity rate and ageing rate of the prey population, respectively. $\eta$ and $f$ are the density dependent coefficients of immature prey population and predator population, respectively. $k(0<k<1)$ is the rate of conversing prey into predator and $E$ is the predation coefficient. $\tau$ and $\tau_{1}$ are the gestation delay and density dependent for predator population, respectively. 
Note that in (2.1), $x_{3}(t)$ is linear dependent on $x_{2}(t)$. That is, the asymptotic behavior of $x_{3}(t)$ is dependent on $x_{2}(t)$. Therefore, we just need to study following subsystem

$$
\left\{\begin{array}{l}
x_{1}^{\prime}(t)=\alpha x_{2}(t)-\gamma x_{1}(t)-\eta x_{1}^{2}(t)-E x_{1}(t) y(t), \\
x_{2}^{\prime}(t)=\Omega x_{1}(t)-\theta x_{2}(t), \\
y^{\prime}(t)=y(t)[k E x(t-\tau)-d-f Q(t)], \\
Q^{\prime}(t)=\delta(y(t)-Q(t)),
\end{array}\right.
$$

where

$$
\begin{aligned}
& Q(t)=\int_{-\infty}^{t} y\left(\tau_{1}\right) \delta \mathrm{e}^{-\delta\left(t-\tau_{1}\right)} \mathrm{d} \tau_{1}, \\
& \gamma=\gamma_{1}+\Omega, \theta=\theta_{1}+a .
\end{aligned}
$$

The initial conditions for (2.2) are

$$
\begin{aligned}
& x_{1}(t)=\varphi_{1}(t) \geq 0, x_{2}(t)=\varphi_{2}(t) \geq 0, \\
& y(t)=\varphi_{3}(t) \geq 0, Q(t)=\varphi_{4}(t) \geq 0, t \in[-\tau, 0] .
\end{aligned}
$$

\section{Local Stability Analysis and Hopf Bifurcation}

\subsection{Local Stability Analysis}

Obviously, system (2.2) has two boundary equilibrium $E_{0}=(0,0,0,0), E_{1}\left(x_{1}, x_{2}, 0,0\right)$ (if condition $C_{1}: \alpha \Omega-\gamma \theta>0$ holds), and an unique positive equilibrium $E_{2}\left(x_{1}^{*}, x_{2}^{*}, y^{*}, Q^{*}\right)$ (if condition $C_{2}: k E x_{1}^{*}-d>0$ holds), where

$$
\begin{aligned}
& x_{1}=\frac{\alpha \Omega-\gamma \theta}{\eta \theta}, x_{2}=\frac{\Omega}{\theta} x_{1}, x_{1}^{*}=\frac{f(\alpha \Omega-\gamma \theta)+d E \theta}{\theta\left(k E^{2}+\eta f\right)}, \\
& x_{2}^{*}=\frac{\Omega}{\theta} x_{1}^{*}, y^{*}=\frac{k E x_{1}^{*}-d}{f}, Q^{*}=y^{*} .
\end{aligned}
$$

Let $U(t)=\left(x_{1}(t), x_{2}(t), y(t), Q(t)\right)^{\mathrm{T}}$,

$\bar{E}=\left(\bar{x}_{1}, \bar{x}_{2}, \bar{y}, \bar{Q}\right)$ be any arbitrary equilibrium. The linearized equations are

$$
U^{\prime}(t)=A U(t)+B U(t-\tau)
$$

where

$$
\begin{aligned}
& A=\left[\begin{array}{cccc}
-\gamma-2 \eta \bar{x}_{1}-\bar{y} E & \alpha & -\bar{x}_{1} E & 0 \\
\Omega & -\theta & 0 & 0 \\
0 & 0 & k \bar{x}_{1} E-d-f \bar{Q} & -\bar{y} f \\
0 & 0 & \delta & -\delta
\end{array}\right] \\
& B=\left[\begin{array}{cccc}
0 & 0 & 0 & 0 \\
0 & 0 & 0 & 0 \\
k \bar{y} E & 0 & 0 & 0 \\
0 & 0 & 0 & 0
\end{array}\right],
\end{aligned}
$$

and the characteristic equation about $\bar{E}$ is given by

$$
\operatorname{det}\left(A+B \mathrm{e}^{-\lambda \tau}-\lambda I\right)=0 .
$$

Theorem 1. 1) $E_{0}$ is local stable if $\gamma \theta>\alpha \Omega$, local unstable if $\gamma \theta<\alpha \Omega$ and $E_{1}$ exist.

2) $E_{1}$ is local stable if $k E x_{1}<d$, local unstable if $k E x_{1}>d$ and $E_{2}$ exist.

Proof. 1) From (3.2), the characteristic equation about $E_{0}$ is given by

$$
(\lambda+d)(\lambda+\delta)\left[\lambda^{2}+(\gamma+\theta) \lambda+\gamma \theta-\alpha \Omega\right]=0 .
$$

Then, $\lambda_{3}=-d<0, \lambda_{4}=-\delta<0$, and $\lambda_{1}, \lambda_{2}$ are the two other roots of

$$
\lambda^{2}+(\gamma+\theta) \lambda+\gamma \theta-\alpha \Omega=0 .
$$

By Routh-Hurwitz criterion, $E_{0}$ is local stable if $\gamma \theta>\alpha \Omega$, local unstable if $\gamma \theta<\alpha \Omega$ and $E_{1}$ exist.

2) From (3.2), the characteristic equation about $E_{1}$ is given by

$$
(\lambda+\delta)\left(\lambda+d-k E x_{1}\right)\left[\lambda^{2}+\left(\gamma+\theta+2 \eta x_{1}\right) \lambda+\theta \eta x_{1}\right]=0 .
$$

Then, $\lambda_{1}, \lambda_{2}$ are the two roots of

$$
\lambda^{2}+\left(\gamma+\theta+2 \eta x_{1}\right) \lambda+\theta \eta x_{1}=0,
$$

with negative real parts. $\lambda_{3}=k E x_{1}-d, \lambda_{4}=-\delta<0$, by Routh-Hurwitz criterion, $E_{1}$ is local stable if $k E x_{1}<d$, local unstable if $k E x_{1}>d$ and $E_{2}$ exist.

\subsection{Existence of Local Hopf Bifurcation}

The characteristic equation about the positive equilibrium $E_{2}$ is given by

$$
D(\lambda, \tau)=M(\lambda)+N(\lambda) \mathrm{e}^{-\lambda \tau}=0 .
$$

where

$$
\begin{aligned}
& M(\lambda)=\lambda^{4}+m_{3} \lambda^{3}+m_{2} \lambda^{2}+m_{1} \lambda+m_{0}, \\
& N(\lambda)=n_{2} \lambda^{2}+n_{1} \lambda+n_{0}, \\
& m_{3}=\delta+\theta+\gamma+2 \eta x_{1}^{*}+E y^{*}, \\
& m_{2}=\theta \eta x_{1}^{*}+\delta\left(\theta+\gamma+2 \eta x_{1}^{*}+E y^{*}+f y^{*}\right), \\
& m_{1}=\delta\left[\theta \eta x_{1}^{*}+f y^{*}\left(\theta+\gamma+2 \eta x_{1}^{*}+E y^{*}\right)\right], \\
& m_{0}=\delta f y^{*} \theta \eta x_{1}^{*}, n_{2}=k E^{2} x_{1}^{*} y^{*} \\
& n_{1}=k E^{2} x_{1}^{*} y^{*}(\delta+\theta), n_{0}=k E^{2} x_{1}^{*} y^{*} \delta \theta,
\end{aligned}
$$

When $\tau=0,(3.5)$ becomes to

$$
\lambda^{4}+m_{3} \lambda^{3}+\left(m_{2}+n_{2}\right) \lambda^{2}+\left(m_{1}+n_{1}\right) \lambda+m_{0}+n_{0}=0 .
$$

and $m_{3}>0, m_{2}+n_{2}>0, m_{1}+n_{1}>0, m_{0}+n_{0}>0$. Note that

$$
\begin{aligned}
& \left(m_{2}+n_{2}\right) m_{3}-\left(m_{1}+n_{1}\right)>0, \\
& \left(m_{1}+n_{1}\right)\left(m_{2}+n_{2}\right) m_{3}-\left(m_{1}+n_{1}\right)^{2}-\left(m_{0}+n_{0}\right) m_{3}^{2}>0, \\
& \left(m_{0}+n_{0}\right)\left[\left(m_{1}+n_{1}\right)\left(m_{2}+n_{2}\right) m_{3}-\left(m_{1}+n_{1}\right)^{2}-m_{3}^{2}\right]>0,
\end{aligned}
$$


if condition $C_{3}: f \eta<k E^{2}$ holds. By Routh-Hurwits criterion, all roots of (3.6) have negative real parts. Then, the equilibrium $E_{2}$ is local stable.

Suppose $\lambda=\mathrm{i} \omega, \omega>0$ is a root of (3.5) and separating the real and imaginary parts, one can get that

$$
\left\{\begin{array}{l}
\omega^{4}-m_{2} \omega^{2}+m_{0}+\left(n_{0}-n_{2} \omega^{2}\right) \cos \omega \tau+n_{1} \omega \sin \omega \tau=0, \\
m_{1} \omega-m_{3} \omega^{3}+n_{1} \omega \cos \omega \tau-\left(n_{0}-n_{2} \omega^{2}\right) \sin \omega \tau=0 .
\end{array}\right.
$$

From (3.7), we have

$$
\begin{aligned}
& \left(n_{0}-n_{2} \omega^{2}\right)^{2}+\left(n_{1} \omega\right)^{2} \\
& =\left(\omega^{4}-m_{2} \omega^{2}+m_{0}\right)^{2}+\left(-m_{3} \omega^{3}+m_{1} \omega\right)^{2},
\end{aligned}
$$

namely

$$
F(\varpi)=\varpi^{4}+f_{3} \varpi^{3}+f_{3} \varpi^{2}+f_{1} \varpi+f_{0}=0
$$

where

$$
\begin{aligned}
& \varpi=\omega^{2}, f_{3}=m_{3}^{2}-2 m_{2}-n_{3}^{2}, \\
& f_{2}=m_{2}^{2}+2 m_{0}-2 m_{1} m_{3}-n_{2}^{2}+2 n_{1} n_{3}, \\
& f_{1}=m_{1}^{2}-2 m_{0} m_{2}+2 n_{0} n_{2}-n_{1}^{2}, f_{0}=\left(m_{0}^{2}-n_{0}^{2}\right) .
\end{aligned}
$$

If condition $C_{3}: f \eta<k E^{2}$ hold, then $f_{0}<0$, (3.9) have at least one positive root. Without loss of generality, we assume (3.9) have four distinct positive roots $\varpi_{j}(j=1,2,3,4)$, then (3.5) have four pair of imaginary roots $\pm \omega_{j} \mathrm{i}= \pm \sqrt{\varpi_{j}} \mathrm{i}$. From (3.7), we have

$$
\cos \omega_{j} \tau=\frac{\left(\omega_{j}^{4}-m_{2} \omega_{j}^{2}\right)\left(n_{0}-n_{2} \omega_{j}^{2}\right)+n_{1} \omega_{j}^{2}\left(m_{1}-m_{3} \omega_{j}^{2}\right)}{\left(n_{0}-n_{2} \omega_{j}^{2}\right)^{2}+\omega_{j}^{2} n_{1}^{2}},
$$

Thus, the $\tau_{j n}(n=0,1,2, \cdots)$ corresponding to $\omega_{j}$ are given by

$$
\begin{aligned}
\tau_{j n}= & \frac{1}{\omega_{j}}\left[\frac{\left(\omega_{j}^{4}-m_{2} \omega_{j}^{2}\right)\left(n_{0}-n_{2} \omega_{j}^{2}\right)+n_{1} \omega_{j}^{2}\left(m_{1}-m_{3} \omega_{j}^{2}\right)}{\left(n_{0}-n_{2} \omega_{j}^{2}\right)^{2}+\omega_{j}^{2} n_{1}^{2}}\right] \\
& +\frac{2 n \pi}{\omega_{j}},
\end{aligned}
$$

And the direction of $\tau_{j n}$ pass through the imaginary axis [8] when $\omega=\omega_{j}$ is given by

$$
\begin{aligned}
& \operatorname{sign}\left[\left.\frac{\mathrm{d} F\left(\omega^{2}\right)}{\mathrm{d} \omega}\right|_{\omega=\omega_{j}}\right] \\
& =\operatorname{sign}\left[2 \omega\left(4 \omega^{6}+3 f_{3} \omega^{4}+2 f_{2} \omega^{2}+f_{1}\right)\right] \\
& =\operatorname{sign}(\Delta) .
\end{aligned}
$$

Then $\operatorname{sign}(\Delta) \neq 0$, since $\varpi_{j}(j=1,2,3,4)$ four distinct positive roots of (3.9). Let

$$
\tau_{0}=\min \left\{\tau_{j n}\right\}, j=1,2,3,4 ; n=0,1,2, \cdots .
$$

According to the Hopf bifurcation theorem for functional differential equations [9], (2.2) can undergoes a Hopf bifurcation at the positive equilibrium $E_{2}$ when $\tau=\tau_{0}$. Furthermore, if condition $C_{4}: f_{j}>0(j=1,2,3)$, $f_{0}<0$ holds, then (3.9) have unique positive root $\omega$, and the $\tau_{n}(n=0,1,2, \cdots)$ corresponding to $\omega$ are given by

$$
\begin{aligned}
\tau_{n}= & \frac{1}{\omega}\left[\frac{\left(\omega^{4}-m_{2} \omega^{2}\right)\left(n_{0}-n_{2} \omega^{2}\right)+n_{1} \omega^{2}\left(m_{1}-m_{3} \omega^{2}\right)}{\left(n_{0}-n_{2} \omega^{2}\right)^{2}+\omega^{2} n_{1}^{2}}\right] \\
& +\frac{2 n \pi}{\omega},
\end{aligned}
$$

and $\operatorname{sign}(\Delta)=1$, according to the Hopf bifurcation theorem for functional differential equations [9], (2.2) can undergoes a Hopf bifurcation at the positive equilibrium $E_{2}$ when $\tau=\tau_{n}(n=0,1,2, \cdots)$. Based on above analysis, we have the following result.

Theorem 2. 1) If condition $C_{3}: f \eta<k E^{2}$ holds, then there exists a $\tau_{0}$, when $\tau \in\left[0, \tau_{0}\right)$ the positive equilibrium $E_{2}$ of (1.2) is asymptotically stable and unstable when $\tau>\tau_{0}$, where $\tau_{0}$ is defined by (2.11).

2) If condition $C_{4}: f_{j}>0(j=1,2,3), f_{0}<0$ holds, then there exists a $\tau_{0}$, when $\tau \in\left[0, \tau_{0}\right)$ the positive equilibrium $E_{2}$ of (2.2) is asymptotically stable and unstable when $\tau>\tau_{0},(2.2)$ can undergoes a Hopf bifurcation at the positive equilibrium $E_{2}$ when $\tau=\tau_{n}(n=0,1,2, \cdots)$, where $\tau_{n}$ is defined by (3.12).

Remark 1. It must be pointed out that Theorem 2 can not determine the stability and the direction of bifurcating periodic solutions, that is, the periodic solutions may exists either for $\tau>\tau_{0}$ or for $\tau<\tau_{0}$, near $\tau_{0}$. To determine the stability, direction and other properties of bifurcating periodic solutions, the normal form theory and center manifold argument should be considered [10].

\section{Numerical Simulation}

We consider following stage-structured delay system

$$
\left\{\begin{array}{l}
x_{1}^{\prime}(t)=3.3 x_{2}(t)-1.04 x_{1}(t)-0.81 x_{1}^{2}(t)-1.05 x_{1}(t) y(t), \\
x_{2}^{\prime}(t)=0.91 x_{1}(t)-0.67 x_{2}(t), \\
y^{\prime}(t)=y(t)[0.7455 x(t-\tau)-0.16-0.23 Q(t)], \\
Q^{\prime}(t)=2.4(y(t)-Q(t)),
\end{array}\right.
$$

where

$$
\begin{aligned}
& \alpha=3.3, \gamma_{1}=0.13, \Omega=0.91, \eta=0.81, E=1.05, \\
& \theta_{1}=0.25, a=0.42, k=0.71, d=0.16, f=0.23, \\
& \delta=2.4, X(0)=(2,2,2,2) .
\end{aligned}
$$


System (4.1) has an unique positive equilibrium point $E_{2}=(0.9903,1.3450,2.5142,2.5142)$. We solve model (4.1) using function dde23 in MATLAB, and compute that

$$
\begin{aligned}
& f_{3}=37.3624, f_{2}=101.0105, f_{1}=43.4939, \\
& f_{0}=-9.2655, \omega_{0}=0.3930, \tau_{0}=4.5113 .
\end{aligned}
$$

According to Theorem 2, the positive equilibrium point $E_{2}$ is asymptotically stable when $\tau=4.4<\tau_{0}$ (see Figure 1). When $\tau=4.6>\tau_{0}$, the positive equilibrium point $E_{2}$ is unstable and the Hopf bifurcation occurring around the positive equilibrium $E_{2}$ are shown (see Figure 2). The bifurcating periodic solution (limit cycle) of (4.1) are stable when $\tau=5,7,10,20,30,40$ (see Figure 3), the amplitudes of period oscillatory are increasing as time delays increased. But, too large time delay would make the population to be die out, because the population very close to zero as time delay increase to some critical value.

\section{Conclusion}

In this paper, we considered a three-stage-structured prey-predator system with discrete and continuous time delays and analyzed the stability of the boundary and positive equilibrium, obtained the conditions of the positive equilibrium occurring Hopf bifurcation by analyzing the characteristic equation about it. Numerical examples by time-series plot have shown that the system considered local asymptotically stable when $\tau<\tau_{0}$ and stable Hopf bifurcation periodic solutions when $\tau>\tau_{0}$ and $\tau$ near $\tau_{0}$. That is to say, time delay can make the positive equilibrium lose stability. It is shown that populations can be coexistence with periodic fluctuating under some conditions and such fluctuation is caused by the time delay. The bifurcating periodic solution (limit cycle) is stable when $\tau$ from 5 to 40 and the amplitudes of period oscillatory are increasing as time delays increased. But, too large time delay would make the population to
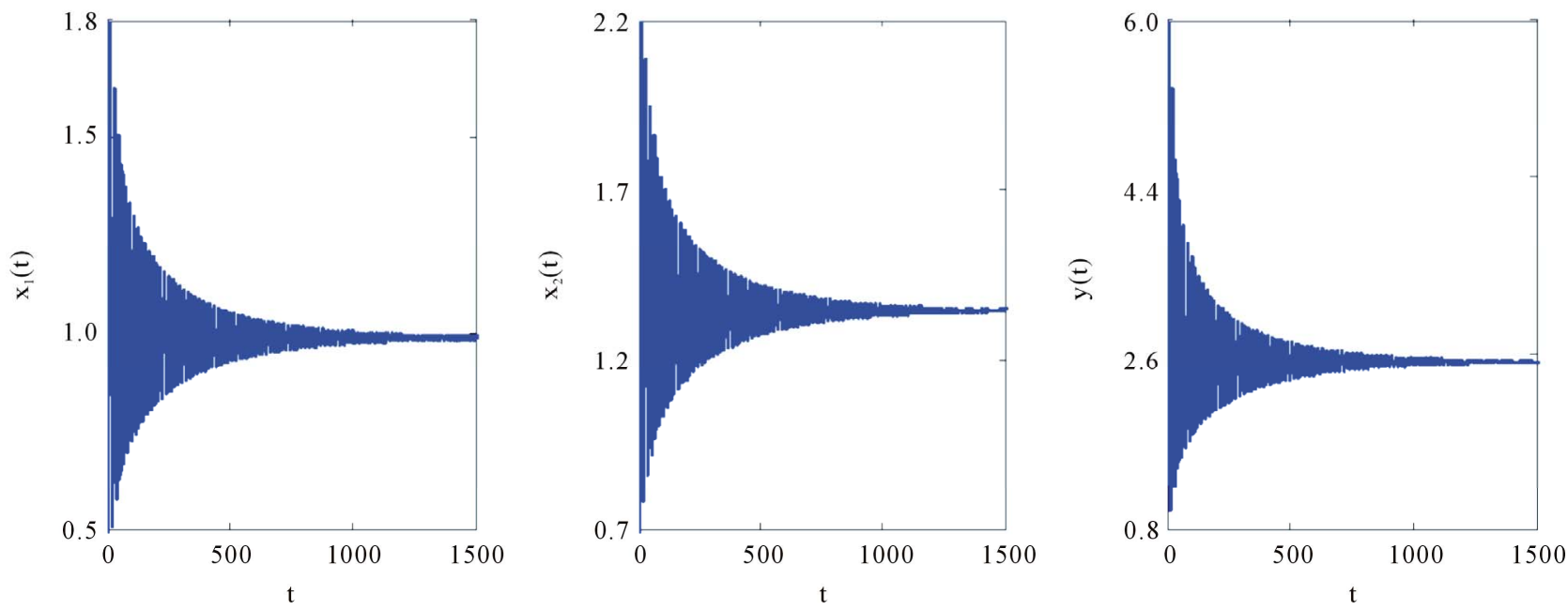

Figure 1. The time-series plot show that positive equilibrium point $E_{2}$ of (4.1) are asymptotically stable for $\tau=4.4<\tau_{0}$.
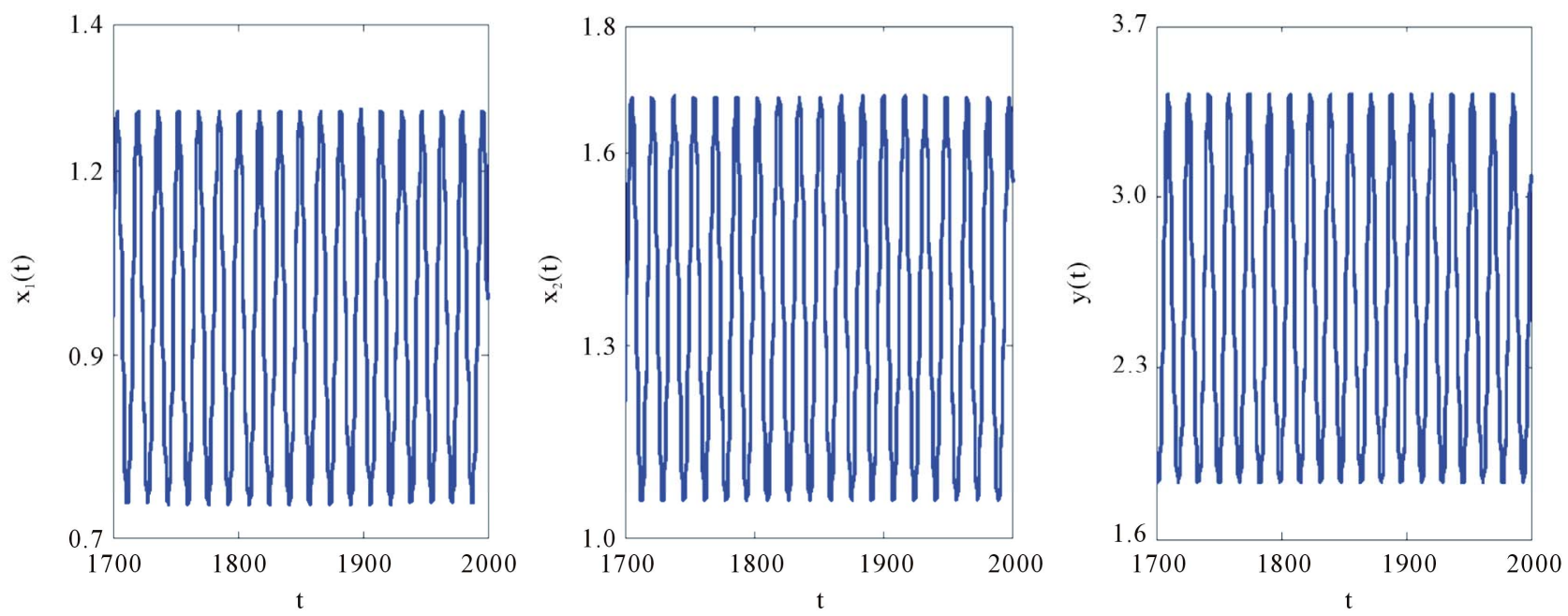

Figure 2. The time-series plot show that (4.1) undergoes Hopf bifurcation for $\tau=4.6>\tau_{0}$. 

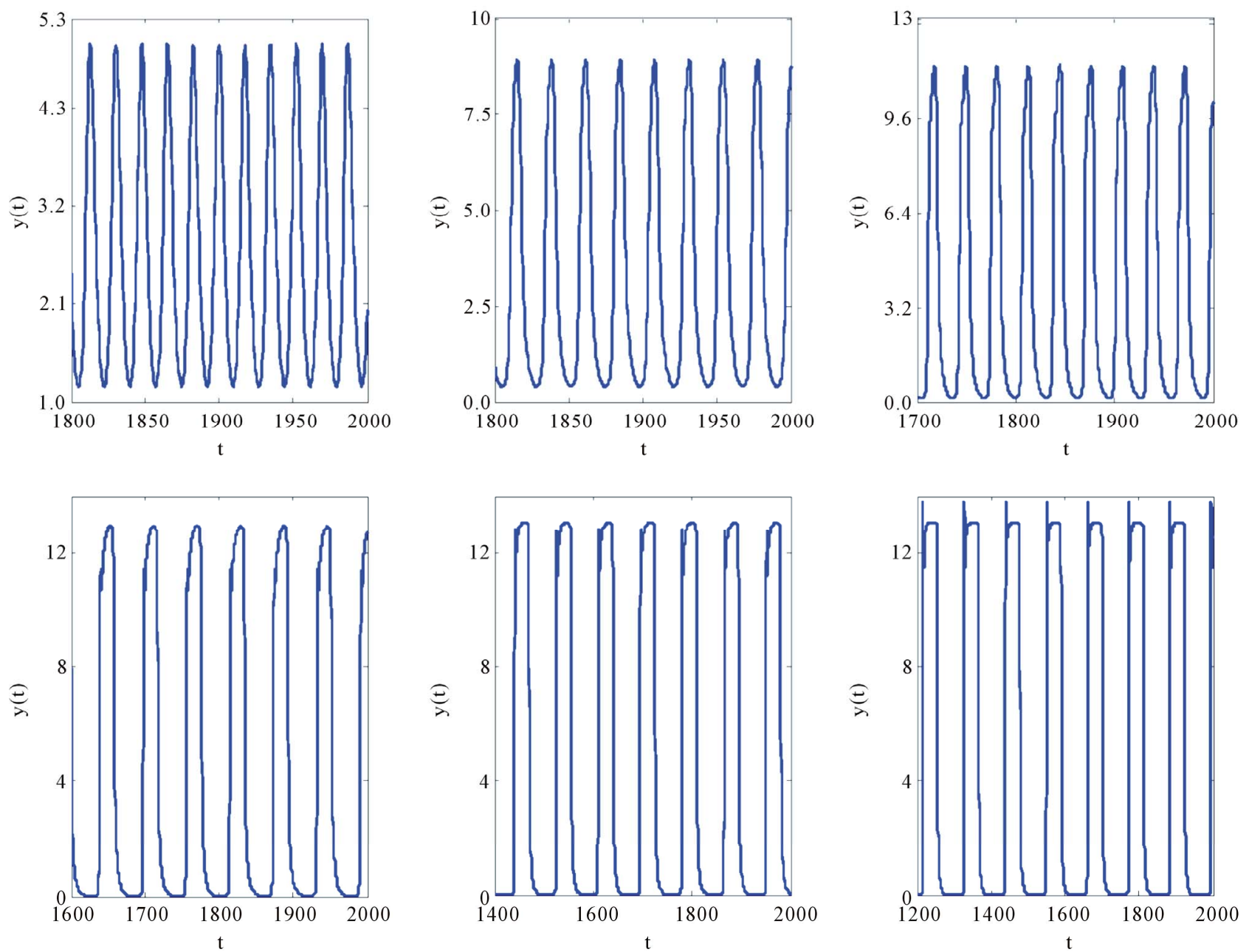

Figure 3. The Hopf bifurcating periodic solution (limit cycles) of (4.1) when $\tau=5,7,10,20,30,40$.

be extinct, because the population arbitrary close to zero as time delay increase to some critical value. These are very interesting in mathematics and biology.

\section{Acknowledgements}

This work was supported by the Natural Science Foundation of Guizhou Province (No. [2011] 2116).

\section{REFERENCES}

[1] W. Aiello and H. Freedman, "A Time-Delay Model of Single-Species Growth with Stage Structure," Mathematical Biosciences, Vol. 101, No. 2, 1990, pp. 139-153. doi:10.1016/0025-5564(90)90019-U

[2] W. Wang and L. Chen, "A Predator-Prey System with Stage-Structure for Predator," Computers \& Mathematics with Applications, Vol. 33, No. 8, 1997, pp. 83-91. doi:10.1016/S0898-1221(97)00056-4

[3] S. Liu, L. Chen and R. Agarwal, "Recent Progress on Stage Structured Population Dynamics," Mathematical and Computer Modelling, Vol. 36, No. 11-13, 2002, pp.
1319-1360. doi:10.1016/S0895-7177(02)00279-0

[4] S. Gao, "Global Stability of Three-Stage-Structured Single-Species Growth Model," Journal Xinjiang University, Vol. 18, No. 12, 2001, pp. 154-158 (in Chinese).

[5] S. Yang and B. Shi. "Periodic Solution for a Three-StageStructured Predator-Prey System with Time Delay," Journal of Mathematical Analysis and Applications, Vol. 341, No. 1, 2008, pp. 287-294. doi:10.1016/j.jmaa.2007.10.025

[6] S. Li and X. Xue, "Hopf Bifurcation in a Three-StageStructured Prey-Predator System with Predator Density Dependent," Communications in Computer and Information Science, Vol. 288, 2012, pp. 740-747. doi:10.1007/978-3-642-31965-5_86

[7] S. Li, Y. Xue and W. Liu, "Hopf Bifurcation and Global Periodic Solutions for a Three-Stage-Structured PreyPredator System with Delays," International Journal of Informationa and Systems Sciences, Vol. 8, No. 1, 2012, pp. 142-156.

[8] Z. Wang, "A Very Simple Criterion for Characterizing the Crossing Direction of Time-delayed Systems with Delay-Dependent Parameters," International Journal of 
Bifurcation and Chaos, Vol. 22, No. 3, 2012, Article ID: 1250048. doi:10.1007/978-1-4612-9892-2

[9] J. Hale, "Theory of Functional Differential Equations," Springer, New York, 1977.
[10] B. Hassard, N. Kazarinoff and Y. Wan, "Theory and Applications of Hopf Bifurcation," Cambridge University Press, Cambridge, 1981. 